\title{
The intimacy of discussion topics: A comparison of three scaling methods
}

\author{
RICHARD C. SHERMAN and JOHN L. GOODSON \\ Miami University, Oxford, Ohio 45056
}

\begin{abstract}
Twenty discussion topics were scaled for intimacy of content using (a) category ratings, (b) Thurstone pair-comparison procedures, and (c) the method of magnitude estimation. The relationship between the magnitude values and both the category ratings and Thurstone values was curvilinear, such that stimulus differences in intimacy were larger at the low end of the continuum and smaller at the high end relative to magnitude scale differences. The implications of these results for research in the area of personal disclosure are discussed in terms of the differences in conclusions which might be reached depending upon the method used to assess the intimacy of disclosed material.
\end{abstract}

In recent years an increasing amount of theory and research has focused upon self-disclosure as it both affects and is affected by various factors in dyadic relationships (Cozby, 1972; Doster \& Strickland, 1969; Halverson \& Shore, 1969; Jourard, 1969). Cozby (1973) has suggested that the basic parameters of self-disclosure are (a) amount of information disclosed, (b) degree of intimacy of the information, and (c) duration of the disclosures. The measurement of amount and duration is relatively straightforward and poses few problems for investigators. However, the intimacy parameter, though it is perhaps the most important of the three, is the most difficult to assess, and its measurement is still most open to question. The adequacy of previous attempts to measure intimacy is the major concern of the present investigation.

The techniques for generating intimacy scale values have included simply averaging judges' category ratings (e.g., Doster \& Strickland, 1971) as well as more complex methods of Thurstone scaling (Taylor \& Altman, 1966; Worthy, Gary, \& Kahn, 1969). In other fields of research, where a physical counterpart to the judgment scale exists, category ratings and Thurstone scales have been found to be affected by the discriminability of stimuli at different points along the physical continuum (Stevens, 1971; Stevens \& Glanater, 1957), as well as by other factors, such as the number of judgment categories allowed to subjects (Marks, 1968). As a result of these factors, intervals at one point on the continuum may not be equal to the same intervals at another point. Although Marks (1968) has shown that this problem may be at least partially overcome by increasing the number of rating categories, disclosure researchers have never used response scales with more than 6 to 12 steps.

Requests for reprints should be addressed to the first author, Department of Psychology, Miami University, Oxford, Ohio 45056. The second author is now at Rutgers University. This paper is sponsored by John C. Jahnke, who takes full editorial responsibility for its contents.
In psychophysics several alternatives to category ratings and Thurstone scaling procedures have been proposed to avoid the above problems, but the technique which has received perhaps the most widespread use to date is the method of magnitude estimation developed by S.S. Stevens. Although magnitude estimation has not been previously used in the scaling of intimacy, it has been applied to several other continua (e.g., strength of religious attitudes, seriousness of crimes, and aesthetic judgments) which are similar to intimacy in that they do not have "physical" counterparts (Stevens, 1966, 1972).

Because of the differences between magnitude and both rating and Thurstone scales in the sizes of subjective intervals along the continuum, the relationship between them is very often nonlinear (cf. Cliff, 1973; Marks, 1974; Stevens, 1966). Further, Stevens (1972) had demonstrated that the nonlinear relationship between magnitude and other scales holds both for attributes which have physical counterparts and those which do not. It is this possible nonlinearity which justifies the attention of disclosure researchers, since they have relied heavily upon both the position of stimuli and separation between stimuli along an intimacy continuum. This reliance is particularly crucial, for example, in studies which have examined the form of the relationship between initial and reciprocated disclosures (e.g., (Cozby, 1972; Worthy, Gary, \& Kahn, 1969). Since the relationship between magnitude and other scales is generally concave downward when they are plotted against the magnitude scale, this implies that differences in intimacy values may be overestimated at the low end of the continuum and underestimated at the high end relative to magnitude scale differences. Depending upon the degree of curvature, then, quite different results might be obtained when these values are used to construct levels of treatments or as measures of treatment effects. 
Table 1

Intimacy Scale Values for 20 Discussion Topics

\begin{tabular}{lccr}
\hline & \multicolumn{3}{c}{ Scale } \\
Topic & Thurstone & Category & Magnitude \\
\hline Weather & 1.00 & 1.41 & 1.12 \\
Food & 1.85 & 1.66 & 12.64 \\
Movies & 1.86 & 1.83 & 14.27 \\
Clothes & 1.88 & 2.00 & 18.56 \\
Politics & 2.11 & 2.52 & 22.72 \\
Hometown & 2.32 & 2.28 & 30.24 \\
Hobbies & 2.44 & 2.03 & 32.85 \\
Financial status & 2.99 & 6.02 & 50.63 \\
Vocational plans & 3.00 & 3.27 & 32.17 \\
Social habits & & & \\
$\quad$ (smoking, drinking) & 3.04 & 4.11 & 28.98 \\
Personal opinion on & & & \\
$\quad$ social issues & 3.12 & 3.63 & 53.50 \\
Dating & 3.52 & 3.88 & 79.72 \\
Religion & 3.83 & 4.09 & 62.12 \\
Death & 3.87 & 5.05 & 91.47 \\
Mutual friends (problems, & & & \\
$\quad$ behavior traits) & 4.07 & 5.97 & 105.44 \\
Personal goals & 4.08 & 4.31 & 69.87 \\
Marriage & 4.43 & 5.42 & 133.12 \\
Personal sex life & 4.87 & 7.94 & 160.81 \\
Personal inadequacies & 4.97 & 6.95 & 147.04 \\
Family problems & 5.03 & 7.58 & 125.97 \\
\hline
\end{tabular}

The purpose of the present study was to obtain category, Thurstone, and magnitude scale values on an intimacy continuum for the same set of stimuli and to examine the functions relating the three sets of values in order to determine the substantive differences which might arise in using the different scales in disclosure research. Discussion topics were selected as stimuli because many previous substantive investigations have used similar materials (cf. Cozby, 1973), thus making the present results applicable to a sizeable body of research.

\section{METHOD}

\section{Subjects}

A total of 166 introductory psychology students participated for course credit in the judgment conditions of the study, and an additional eight undergraduates aided in the generation of the initial pool of discussion topics. Approximately equal numbers of males and females participated in both the topic generation and judgment phases.

\section{Stimuli}

An initial list of 26 discussion topics was constructed by having the small group of undergraduate men and women generate what they felt were appropriate examples of Rubin's (1974) five categories of self-disclosure, ranging from "little or no self-disclosure" to "intimate disclosure." Following the elimination of ambiguous and redundant items, a final set of 20 topics, four from each category, was selected for the judgment tasks.

\section{Procedure}

Subjects were run in groups of 20 to 30 . All members of a group were assigned either to (1) a pair-comparison judgment condition (49 subjects total), (2) a category rating condition (64 subjects total), or (3) a magnitude estimation condition (53 subjects total).
In the pair-comparison condition, the topics were randomly ordered and the 190 distinct pairs were presented to each subject on numbered cards, one for each topic pair. Subjects were instructed to indicate which topic of each pair was more intimate and to record their judgments on an answer sheet which contained a lower half-matrix of numbered cells corresponding to the topic pairs. The order of the 190 stimulus cards was randomized differently for each subject.

In the category rating condition, subjects rated each topic on a scale from 1 (not at all intimate) to 9 (very intimate). All subjects rated the topics in the same randomized order.

In the magnitude estimation task, the topics were randomly numbered and presented on cards, one for each topic. Subjects received instructions adapted from standard psychophysical instructions for "modulus-free" magnitude estimation (cf. Engen, 1971). Following suggested procedures by Stevens (1966), subjects were first familiarized with the magnitude estimation procedure by giving them two examples of estimating lengths of lines. Also, the first topic for all subjects was from Rubin's "moderate" category, although the order for the remaining topics was randomized differently for each subject.

\section{RESULTS AND DISCUSSION}

\section{Scale Values}

Thurstone Scale. The Thurstone scale values were determined from the pair-comparison data according to Thurstone's "Case V" assumptions (Engen, 1971; Thurstone, 1972). The resulting values are given in the first column of Table 1.

Category Rating and Magnitude Scale Values. The 9-point intimacy ratings for each topic were arithmetically averaged across subjects to obtain the category scale values. These are shown in the second column of Table 1. The magnitude estimation scale was constructed by computing the geometric means of subjects' responses to each discussion topic (Marks, 1974; Stevens, 1971). The magnitude values are given in the last column of Table 1 .

Relationships Between the Scales. In order to examine the relationships between the magnitude and both the Thurstone and category scales, it was desirable to specify a function which could represent a wide range of possible relationships, varying from concave downward, to linear, to concave upward (when the Thurstone or category values were plotted against the magnitude values). A power function of the form

$$
\Psi_{1}=\mathrm{a} \Psi_{2}^{\mathrm{b}}
$$

has these properties and has been previously used for relating the magnitude scale values to other response scales (cf. Marks, 1968, 1974). In Equation 1, $\Psi_{1}$ represents either the category or Thurstone scale value of a topic, a is a scaling constant, $\Psi_{2}$ is the magnitude scale value of a topic, and $b$ is a positive exponent. When $\mathrm{b}=1$, the relationship between $\Psi_{1}$ and $\Psi_{2}$ is linear. When $b<1$, the relationship is concave downward, and concave upward when $b>1$.

In the present problem two questions are of interest. 
First, regardless of the form of the relationship, how well do the data fit the obtained function? Second, does the obtained function differ significantly from linearity and, if so, in what direction? Taking the logarithms of both sides of Equation 1 reduces these questions to a simple matter of least squares regression, where

$$
\log \Psi_{1}=\log \mathrm{a}+\mathrm{b} \log \Psi_{2}
$$

and $\mathrm{b}$ becomes the regression coefficient and $\log \mathrm{a}$ the regression constant. Standard procedures may then be used to test the significance of the regression. Also, the value of $b$ may be tested against the linear null hypothesis $(b=1)$ with the statistic

$$
\mathrm{t}=\frac{1-\mathrm{b}}{\mathrm{S}_{\mathrm{b}}}
$$

where $\mathrm{S}_{\mathrm{b}}$ is the standard error of the regression coefficient (Hald, 1952). In the present study the alternative hypotheses are directional $(b<1)$, since the relation between magnitude and the other types of scale values has most often been found to be concave downward.

The Thurstone and category scale values are shown plotted against the magnitude values in Figure 1. In both cases the regressions for the power functions are significant at $\mathrm{p}<.01(\mathrm{R}=.97$ and .87 for the Thurstone and category scales, respectively). Also, the exponents of .35 (Thurstone) and .40 (category) are both significantly $(\mathrm{p}<.01)$ less than $1.0(\mathrm{t}=29.68$ and 11.06 , respectively, with $18 \mathrm{df}$ ). In other words, both the Thurstone and category scale values can be closely estimated from the magnitude values, but there is a significant curvature to the best-fitting regression line. An examination of the power function in Figure 1 reveals that this is somewhat more true of the Thurstone values than the category scale values. Despite this difference, the category and Thurstone values were highly correlated $(r=.93)$.

\section{Implications and Conclusions}

The exponents of .35 and .40 indicate that at the low end of the continuum, the Thurstone and category values of subjective intimacy grow more rapidly than the magnitude values, whereas at the high end they increase less rapidly. That is, differences in perceived intimacy are greater on the category and Thurstone scales than on the magnitude scale for less intimate topics. For highly intimate topics, however, the differences are small relative to the magnitude differences. These results are consistent with the findings of investigations concerning a wide variety of "nonmetric" continua, including the strength of religious attitudes (Stevens, 1966), the affective value of musical selections (Koh, 1965), and the seriousness of criminal offenses (Sellin
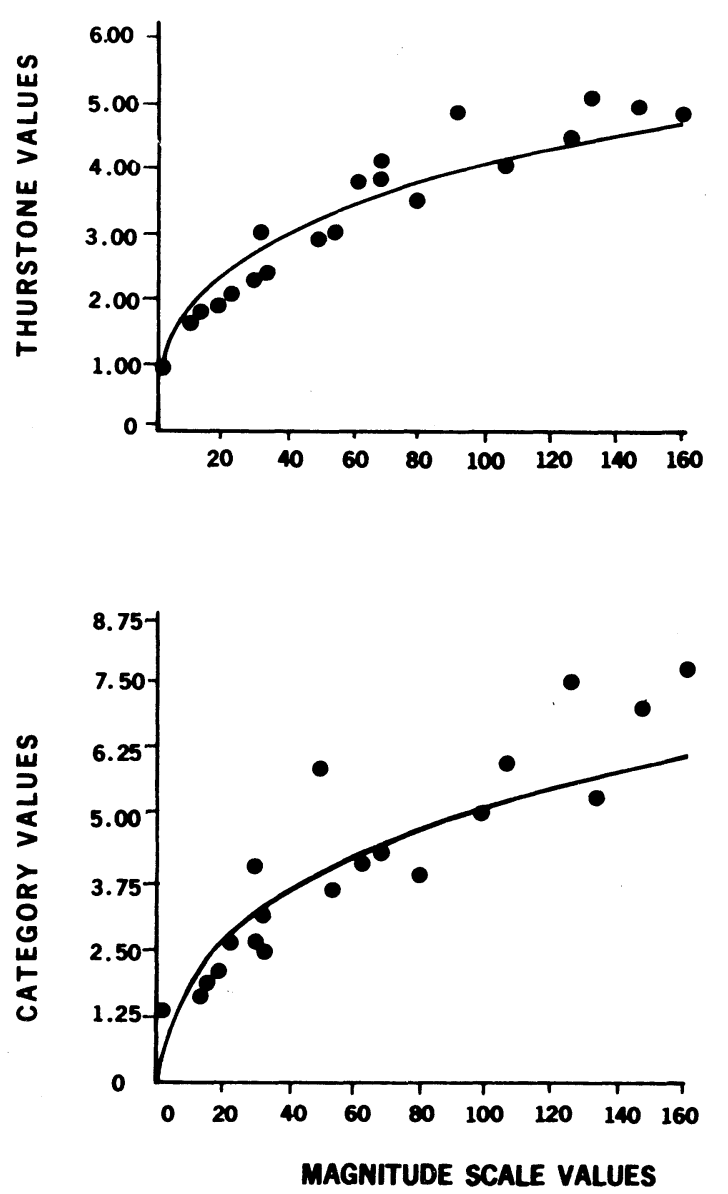

Figure 1. Plots of Thurstone (top) and category (bottom) scales against magnitude values showing the best-fitting power functions. In both cases the functions are significantly nonlinear.

\& Wolfgang, 1964). The question remains, of course, as to what kinds of substantive differences would result from using the magnitude scale instead of either the category or Thurstone scale in disclosure research.

Perhaps the clearest illustration of how these results might bear upon research in the area of disclosure is in an examination of the stimuli which might be selected in an experiment and the differences in conclusions which would follow depending upon the scaling procedure. For example, assume that an investigator wishes to determine the degree to which interpersonal attraction is affected by the intimacy of verbal material that one person discloses to another during an initial encounter. To accomplish this, the experimenter might arrange for a "bogus" stranger to disclose material of low, medium, and high levels of intimacy to subjects and then assess their liking for the stranger. If the Thurstone scale values were used as the basis for selection, likely categories of information might be the weather (1.00), vocational plans (3.00), and family problems (5.03) for the low, medium, and highly intimate conditions. However, the magnitude values 
$(1.12,32.17,125.97)$ imply that the subjective intervals separating these topics are not at all equal, but rather the difference between vocational plans and the weather $(32.17-1.12)$ is only about one-third the difference between family problems and vocational plans (125.97 - 32.17). Thus, it could be argued that the treatment conditions do not differ by a constant amount; subjects in the high vs. medium conditions would actually receive disclosures that were much more discrepant in intimacy than the disclosures received by the low vs. medium groups. The impact of these unequal differences on the investigator's conclusions would depend upon the specific outcome of the experiment, but in general any statement concerning the form of the relationship between attraction and intimacy of disclosures would be mitigated by the fact that the stimulus material was perhaps not evenly sampled from the intimacy continuum as the researcher believed. In particular, the "medium" information would not be "medium" in the sense of being midway in intimacy between the low and high information. Thus, if the investigator found that the different levels of information produced equal increments in liking for the stranger, it would be questionable to conclude that the relationship was linear. In fact, if the magnitude scale values were used in the interpretation of these results, the conclusion would favor the notion that there was a leveling off of the affect of disclosure on liking as the information became more intimate. This conclusion would follow because the increase in intimacy from "medium" to "high" was actually three times greater than the increase from "low" to "medium," yet both increases produced the same increment in liking. Of course, a more thorough representation of intimacy levels would be necessary to determine the precise form of the relationship. It should be noted that both linear and curvilinear relationships between liking and disclosure have been reported in the literature (cf. Cozby, 1973). These discrepancies might be at least partially resolved by giving careful attention to measurement procedures.

As a final note, it should be pointed out that there are several alternatives to the measurement procedures examined here which might be applied to this area. In particular, conjoint measurement (Krantz \& Tversky, 1971) and functional measurement (Anderson, 1970, 1974; Birnbaum, 1974) might lead to a level of refinement in assessing the intimacy of self-disclosure which would allow substantial advances in the sophistication of experimental procedures and therefore the importance of subsequent results. Unfortunately, researchers may tend to avoid such techniques as being too difficult to understand or excessively difficult to apply. It might be well in this regard to offer a hopeful statement by Stevens (1966, p. 538): "Science seems to do its best when it faces a problem worth solving."

\section{REFERENCES}

Anderson, N. H. Cognitive algebra: Integration theory applied to social attribution. In L. Berkowitz (Ed.). Advances in experimental social psychology, Vol. 7. New York: Academic Press, 1974.

ANDERSON, N. H. Functional measurement and psychophysical judgment. Psychological Review, 1970, 77, 153-170.

Birnbaum, M. H. Using contextual effects to derive psychophysical scales. Perception \& Psychophysics, 1974, 15, 89-96.

Cliff, N. Scaling. In P. H. Mussen and M. R. Rosenzweig (Eds.), Annual Review of Psychology. Palo Alto: Annual Reviews, 1973.

Cozby, P. C. Self-disclosure, reciprocity, and liking. Sociometry, $1972,35,151-160$.

Cozby, P. C. Self-disclosure: A literature review. Psychological Bulletin, 1973, 79. 73-91.

Doster, J. A., \& Strickland, B. R. Disclosing of verbal material as a function of information requested, information about the interviewer, and interviewee differences. Journal of Consulting and Clinical Psychology, 1971, 37, 187.

Doster, J. A., \& Strickland, B. R. Perceived child-rearing practices and self-disclosure patterns. Journal of Consulting and Clinical Psychology, 1969, 33, 382.

ENGen, T. Psychophysics: Scaling methods. In J. W. Kling and L. A. Riggs (Eds.), Experimental psychology (3rd ed.). New York: Holt, Rinehart, and Winston, 1971.

HALD, A. Statistical theory with engineering applications. New York: Wiley, 1952.

Halverson, C. F., \& Shore, R. E. Self-disclosure and interpersonal functioning. Journal of Consulting and Clinical Psychology, 1969, 33, 213-217.

JoURARD, S. M. The effects of experimenters' self-disclosure on subjects' behavior. In C. Spielberger (Ed.), Current topics in community and clinical psychology. New York: Academic Press, 1969.

Kон, S. C. Scaling musical preferences. Journal of Experimental Psychology, 1965, 70, 79-82.

Krantz, D. H., \& Tversky, A. Conjoint-measurement analysis of composition rules in psychology. Psychological Review, 1971, 78, 151-169.

Marks, L. E. Stimulus range, number of categories, and form of the category scale. American Journal of Psychology, 1968, 81, 467-479.

MARKs, L. E. Sensory processes: The new psychophysics. New York:Academic Press, 1974.

RuBin, Z. Lovers and other strangers: The development of intimacy in encounters and relationships. American Scientist, $1974,62,82-190$.

SELLIN, T., \& WolfGANG, M. E. The measurement of delinquency. New York: Wiley, 1964.

Stevens, S. S. A metric for the social concensus. Science, $1966,151,530-541$.

Stevens, S. S. Issues in psychophysical measurement. Psychological Review, 1971, 78, 426-450.

Stevens, S. S. Psychophysics and social scaling. Morristown, N.J: General Learning Press, 1972.

Stevens, S. S., \& Galanter, E. H.Ratio scales and category scales for a dozen perceptual continua. Journal of Experimental Psychology, 1957, 54, 377-411.

Taylor, D. A., \& Altman, I. Intimacy-scaled stimuli for use in studies of interpersonal relations. Psychological Reports, 1966,19, 729-730.

Thurstone, L. L. A law of comparative judgment. Psychological Review, 1927, 34, 273-286.

Worthy, M., Gary, A. L., \& KahN, G. M. Self-disclosure as an exchange process. Journal of Personality and Social Psychology, 1969, 13, 59-63.

(Received for publication August 15, 1975.) 